\title{
Beneficios económicos del uso de biodigestores en una empresa productora de quesos
}

Economic benefits of biodigesters use in an enterprise producer of cheese

Ruiz Almazán Ileana ${ }^{1}$, Legarreta-González Martín Alfredo ${ }^{\bowtie 1}$, Briseño García Arturo² ${ }^{2}$ Escalera Ochoa Laura $^{1}$, Castruita Esparza Luis Ubaldo ${ }^{1}$

${ }^{1}$ Facultad de Ciencias Agrícolas y Forestales de la Universidad Autónoma de Chihuahua. Km 2.5 Carretera Delicias-Rosales, Cd. Delicias, Chih. ${ }^{2}$ Facultad de Comercio y Administración Victoria, Universidad Autónoma de Tamaulipas, Cd. Victoria Tamaulipas.

${ }^{凶}$ Autor por correspondencia: mlegarre@uach.mx

Recibido: 01/10/2019

Aceptado: 12/11/2019

\begin{abstract}
RESUMEN
A nivel mundial el uso de combustibles fósiles ha crecido conforme pasa el tiempo. Sin embargo, estos tipos de energía se están agotando, lo que conlleva a que sus costos aumenten, resultado de la oferta y demanda global, por lo que es necesario utilizar otro tipo de energías que no afecten al medio ambiente y tampoco recurran a grandes costos. Una de las maneras de generar energía renovable es a través del uso de biodigestores que permiten la producción de biogás, el cual está compuesto por una mezcla de gases, principalmente dióxido de carbono y metano. Este subproducto puede ser empleado como generador de energía eléctrica o como reemplazo de ciertos combustibles fósiles usados en las actividades diarias. El objetivo del estudio fue calcular el impacto económico generado por la producción y uso de biogás en una empresa productora de quesos en el municipio de Saucillo, Chihuahua, México. Esta empresa cuenta con una instalación de biodigestores, cuya producción de biogás es suficiente para reemplazar el uso del gas LP. En el presente estudio se analizó el impacto económico resultante del uso del proceso de biodigestión en la empresa, a través de diversos indicadores económicos aplicados conforme la información recolectada y de acuerdo a la metodología de Baca Urbina. Obteniendo como resultados una TIR de 46.72\%, VPN de \$4, 937, 745. 64 y una relación beneficio-costo de 1.80. Como conclusión, el uso de los biodigestores proporciona un impacto aceptable y favorable mostrando que la inversión del proyecto es rentable para la empresa.
\end{abstract}

Palabras clave: Impacto económico, beneficios, biogás, reemplazo, rentable

\begin{abstract}
Globally, the use of fossil fuels has increased as time passes, however, these types of energy are depleting, which leads to increased costs, the result of supply and demand. So, it is necessary to use other types of energy that do not affect the environment and do not resort to large costs. One of the ways to generate renewable energy is through the use of biodigesters allowing the production of biogas which is a mixture of gases, mainly carbon dioxide and methane. This by-product can be used as generator of
\end{abstract}


electrical energy or as a replacement for certain fossil fuels used in daily activities. The main objective was to calculate the economic impact generated by the production and use of biogas in a cheese producer company from Saucillo, Chihuahua, México. This enterprise has a biodigester facility, which biogas production is enough to replace its use of LP gas. On the present study is shown the economic impact resulting from the use of the biodigestion process in the company through various economic indicators applied according to the information collected and Baca Urbina methodology. The results obtained were an IRR of $46.72 \%$, NPV of $\$ 4,937,745.64$ and benefit-cost rate of 1.80 . As conclusion the use of biodigesters provide an acceptable and favorable impact showing that the investment of the project is profitable for the company.

Keywords: Economic impact, benefit, biogas, replacement, profitable.

\section{INTRODUCCIÓN}

La energía utilizada en los últimos años, a nivel global, ha sido proporcionada en aproximadamente un $80 \%$ a través del uso de combustibles fósiles, siendo esto producto de la segunda guerra mundial como parte del sistema económico, es decir, se tiene energía más económica y con mayor disponibilidad. Sin embargo, esto ha ocasionado que los recursos sean cada día más escasos y se orille a los seres humanos a conseguir las fuentes de energía necesarias, mediante la explotación de recursos con una menor calidad y mayores costos (Ferrari, 2013).

Después de más de 200 años, donde los combustibles fósiles han predominado, se genera la idea de usar fuentes de energía renovables, que permitan la generación de energía eléctrica o térmica (Marín Quemada \& Martín González, 2018).

Lamentablemente el uso de las energías renovables requiere inversiones iniciales mayores a las requeridas por los combustibles fósiles. Sin embargo, actualmente existen algunas empresas que están optando por realizar inversiones en el sector correspondiente a las energías renovables (Villamil Serrano, 2017).
Una de las maneras que permiten generar energías renovables es la producción de biogás, el cual se define una mezcla de gases, principalmente dióxido de carbono $\left(\mathrm{CO}_{2}\right)$ y metano $\left(\mathrm{CH}_{4}\right)$ (Varnero, 2015), generada mediante un proceso de fermentación microbiana, caracterizada por la ausencia de oxígeno, conocida como biodigestión (Lorenzo \& Obaya, 2005).

Productos Lácteos Los Pinos S.P.R.L. de C.V. es una empresa que produce queso tipo cheddar en la ciudad de Saucillo, Chihuahua y que para aprovechar al máximo de sus recursos y apoyar al medio ambiente, cuenta con la instalación de biodigestores, los cuales, además de los propósitos antes mencionados, producen el biogás suficiente para cubrir las necesidades de la empresa. El objetivo de este documento es mostrar las ventajas, principalmente económicas, que generan el uso de biodigestores en la empresa.

\section{MATERIALES Y MÉTODOS}

El estudio se desarrolló en una empresa productora de quesos que se encuentra en la ciudad de Saucillo, Chihuahua, México. La recopilación de la información se realizó entre septiembre de 2018 y enero de 2019. 
Las variables de estudio fueron los beneficios obtenidos con la producción de biogás, los cuales fueron estimados con el volumen que se generó y el costo del gas LP durante los años analizados, ya que fue el combustible reemplazado por el biogás, así como los costos, tantos variables como fijos, que conlleva el proceso de biodigestión. Los costos fijos están conformados por la mano de obra, tanto directa como indirecta, y los costos variables que incluyen los costos de los servicios empleados en los biodigestores, así como el combustible utilizado para el traslado del estiércol.
El método de recopilación de información fue de mediciones diarias de la producción de biogás y con documentos de registros propiedad de la empresa. Los datos fueron usados para calcular el periodo de retorno, la rentabilidad, Valor Presente Neto (VPN), Tasa Interna de Rendimiento (TIR) y relación costo-beneficio del aprovechamiento del biogás como combustible. Todos estos indicadores fueron calculados tomando como base la sección financiera de la metodología de Baca Urbina (2006), como se muestra en la figura 1.

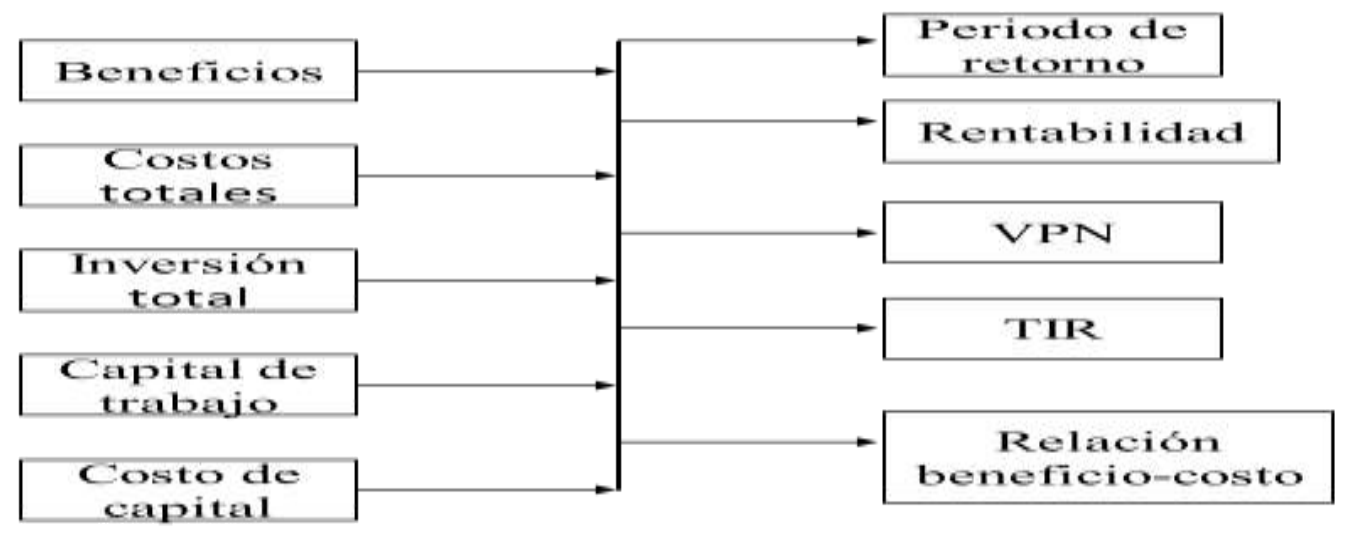

Figura 1. Sección financiera de evaluación de proyectos de acuerdo a Baca Urbina (2006)

\section{RESULTADOS Y DISCUSIÓN}

El análisis de costos se realizó basándose en la información recolectada, así como datos de registro históricos con los que contaba la empresa.

El promedio de producción de biogás diario, de acuerdo con los formatos de registro, es de 927 litros, es decir, se producen aproximadamente 334,050 litros anuales de biogás, el cual es utilizado como reemplazo del gas LP. El costo del gas LP ha sido muy variante en los últimos años, por lo que la cantidad de biogás por año fue multiplicada por el precio del gas LP, de acuerdo a cada uno de los años que se analizaron.

En la figura 2 se muestra la producción promedio de biogás durante el tiempo de medición de variables. 
Ruiz et al., 2019

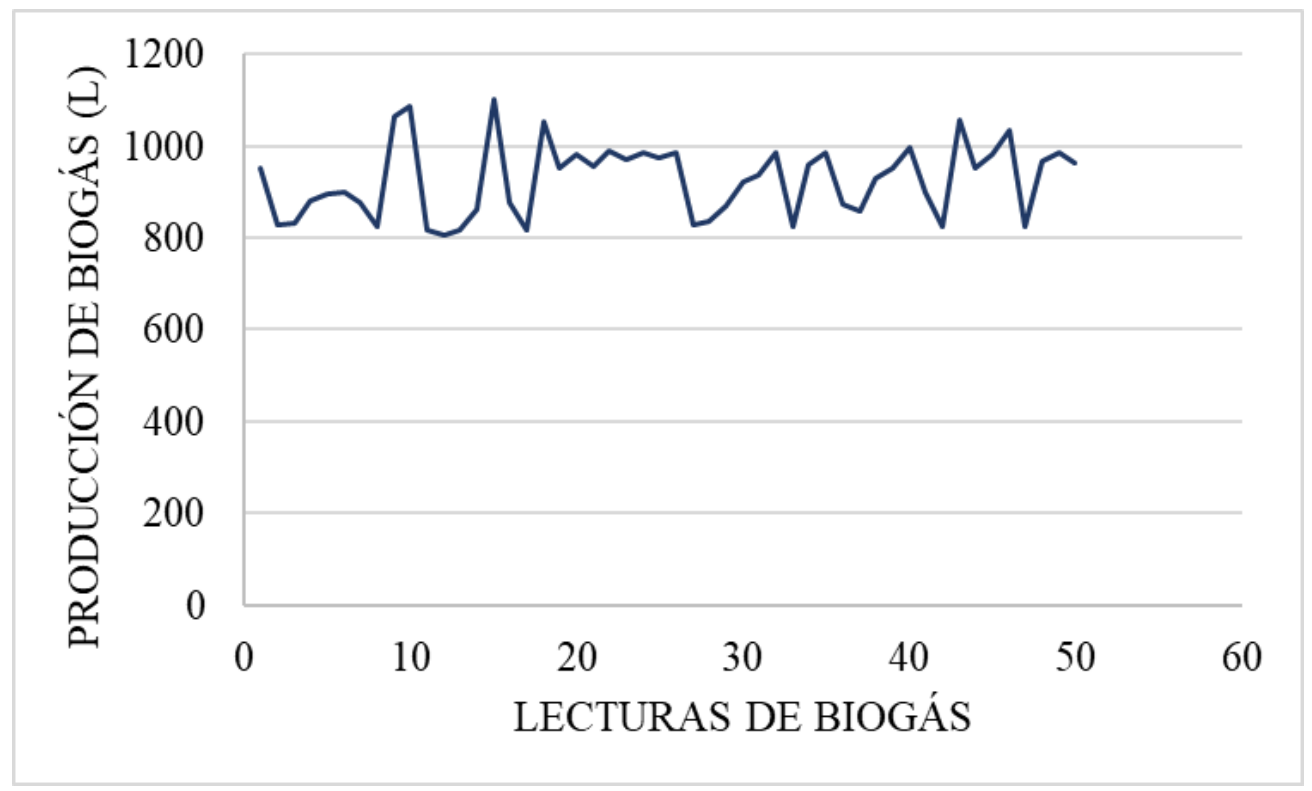

Figura 2. Producción de biogás. Fuente: Elaboración propia con datos tomados en la empresa.

Los costos de inversión fueron desglosados por todas las actividades realizadas para la instalación, los cuales fueron desembolsados desde el inicio del proyecto (año 0).

Los beneficios se estimaron de acuerdo con el impacto que se genera al reemplazar el gas LP con biogás, es decir, el valor del beneficio es igual al costo que la empresa pagaría por consumir el combustible fósil. Además de generar beneficios económicos, el uso de los biodigestores también tiene un impacto en el medio ambiente al reducir los gases de efecto invernadero un $30.49 \%$.

En cuanto a la mano de obra directa, sólo una persona se encarga del cuidado y mantenimiento de los biodigestores directamente, el cual recibe un pago semanal, así como un bono mensual si cumple con los requerimientos de su puesto. Y, como mano de obra indirecta, una parte proporcional del encargado del área de mantenimiento. Siendo estos los costos fijos del área de biodigestores.

Los costos variables incluyen los costos de energía eléctrica, agua, mantenimiento y combustible.

Los flujos de efectivo generados, durante los 5 años, fueron calculados con la diferencia de los beneficios obtenidos y los costos generados en cada uno de los años, como se muestra en el cuadro 1 . 
Ruiz et al., 2019

Cuadro 1. Flujos de efectivo de biodigestores. Fuente: Elaboración propia con información de la empresa.

\begin{tabular}{|c|rr|rr|rr|}
\hline Año & \multicolumn{2}{|c|}{ Beneficios } & \multicolumn{2}{|c|}{ Costos } & \multicolumn{2}{|c|}{ Flujo de efectivo } \\
\hline $\mathbf{0}$ & $\$$ & - & $\$$ & $4,883,958.15$ & $-\$$ & $4,883,958.15$ \\
\hline $\mathbf{1}$ & $\$$ & $2,425,203.00$ & $\$$ & $294,929.30$ & $\$$ & $2,130,273.70$ \\
\hline $\mathbf{2}$ & $\$$ & $2,445,246.00$ & $\$$ & $339,168.70$ & $\$$ & $2,106,077.30$ \\
\hline $\mathbf{3}$ & $\$$ & $3,604,399.50$ & $\$$ & $390,044.00$ & $\$$ & $3,214,355.50$ \\
\hline $\mathbf{4}$ & $\$$ & $4,193,997.75$ & $\$$ & $448,550.60$ & $\$$ & $3,745,447.15$ \\
\hline $\mathbf{5}$ & $\$$ & $4,783,596.00$ & $\$$ & $515,833.19$ & $\$$ & $4,267,762.81$ \\
\hline Total & $\mathbf{\$}$ & $\mathbf{1 7 , 4 5 2 , 4 4 2 . 2 5}$ & $\mathbf{\$} \mathbf{6 , 8 7 2 , 4 8 3 . 9 4}$ & $\mathbf{\$}$ & $\mathbf{1 0 , 5 7 9 , 9 5 8 . 3 1}$ \\
\hline
\end{tabular}

\section{Periodo de retorno}

El periodo de tiempo que le tomó a la empresa recuperar su inversión, de acuerdo a la información recolectada, es de 2 años y 3 meses. Durante los dos primeros años completos, la recuperación de la inversión es de un $99.72 \%$ y el resto se retorna durante los 3 primeros meses de ese segundo año.

\section{Tasa de rentabilidad}

$\$ 6,872,483.94=100 \%$

$\$ 15,463,916.46=X$

Tasa de rentabilidad $=2.25$ o $225 \%$

Es decir, el proyecto es rentable un $125 \%$ más de los costos generados en la inversión. La tasa de rentabilidad es de $225 \%$, tomando en cuenta que la tasa promedio interbancaria actual es de $7.75 \%$. El proyecto ofrece, por mucho, mayores beneficios para la empresa, en el caso de que hubiera decidido usar el capital para otro tipo de proyecto y no instalar los biodigestores.

\section{Valor Presente Neto (VPN)}

Para el cálculo de VPN se incurrió a los flujos de efectivo calculándolos a un valor presente con una tasa de 15\% para después restar la inversión del proyecto la cual fue de $\$ 4,883,958.15$, como se muestra en el cuadro 2 . La tasa de interés fue estimada contemplando la tasa promedio interbancaria en México más la tasa de inflación.

El VPN correspondiente al impacto económico de los biodigestores es de $\$ 4,937,745.64$. 
Cuadro 2. Flujos de efectivo calculados a valor presente. Fuente: Elaboración propia con información de la empresa.

\begin{tabular}{|c|cc|cr|}
\hline Ã்̃ & \multicolumn{2}{|c|}{$\begin{array}{c}\text { Beneficios } \\
\text { Actuales }\end{array}$} & Egresos Actuales \\
\hline $\mathbf{0}$ & $\$$ & - & $\$$ & $4,883,958.15$ \\
\hline $\mathbf{1}$ & $\$$ & $2,108,872.17$ & $\$$ & $256,460.26$ \\
\hline $\mathbf{2}$ & $\$$ & $1,848,957.28$ & $\$$ & $256,460.26$ \\
\hline $\mathbf{3}$ & $\$$ & $2,369,951.18$ & $\$$ & $256,460.26$ \\
\hline $\mathbf{4}$ & $\$$ & $2,397,931.83$ & $\$$ & $256,460.26$ \\
\hline $\mathbf{5}$ & $\$ 2,378,292.64$ & $\$$ & $256,460.26$ \\
\hline Total & $\mathbf{\$ 1 1 , 1 0 4 , 0 0 5 . 1 0}$ & $\mathbf{\$}$ & $\mathbf{6 , 1 6 6 , 2 5 9 . 4 6}$ \\
\hline
\end{tabular}

Al calcular el VPN se obtuvo un resultado de $\$ 4,937,745.64$, siendo esta cantidad, por mucho, mayor a la inversión original, por lo que el proyecto de inversión es aceptable de acuerdo a este indicador. Además, la tasa interna de rendimiento obtenida es mayor que la tasa de capital actual.

\section{Tasa Interna de Rendimiento (TIR)}

La TIR resultante fue de $46.72 \%$ siendo mayor que la tasa de rendimiento deseada (15\%), lo cual demuestra que la inversión realizada es atractiva financieramente, para que el proyecto pueda ser replicado en otras empresas.

\section{Relación beneficio-costo}

La relación beneficio-costo es de 1.80. Por último, la producción y uso de biogás es rentable para la empresa, porque por cada peso que la empresa invirtió en el proyecto, se está ganando $\$ 0.80$.

\section{CONCLUSIONES}

La empresa muestra que la producción de biogás ha sido redituable por el hecho de reemplazar el uso del combustible anteriormente utilizado.

Se encontró que el periodo de retorno es relativamente corto, ya que la recuperación de la inversion es menor a 5 años, a partir de los cuales se obtienen beneficios.

En conclusión, la instalación de los biodigestores es una decisión que ha tenido un impacto relevante en el aspecto económico para la empresa, ya que la recuperación de la inversión ha sido menor de 5 años, en comparación con proyectos que tienen un periodo de recuperación de hasta 10 años. Además, y como punto más importante, el uso mixto de biogás y gas LP como combustibles, lo cual, apoya los gastos y fortalece las finanzas de la empresa. 


\section{LITERATURA CITADA}

Baca Urbina, Gabriel (2006). Evaluación de proyectos. Editorial Mc Graw Hill.

Ferrari, L. (2013). Energías fósiles: diagnóstico, perspectivas e implicaciones económicas. Revista Mexicana de Física, 36-43.

Lorenzo Acosta, Y., \& Obaya Abreu, M. C. (2005). La digestión anaerobia. Aspectos teóricos. Parte I. ICIDCA. Sobre los derivados de la caña de azúcar, 35-48.
Marín Quemada, J. M., \& Martín González, E. S. (2018). El futuro de los combustibles fósiles. (págs. 002-14). Grupo de Investigación Economía Política Internacional.

Varnero Moreno, M. T. (2015). Manual de biogás. Chile: Remoción de barreras para la electrificación rural con energías renovables, 01-120.

Villamil Serrano, A. (2017). Economía y medio ambiente. Barcelona: Editorial Universitaria Ramon Aceres.

Copyright (c) 2019 Ruiz Alm azán Ileana, L egarreta-G onzález Martin Alfredo, Briseño Garcia Arturo, Escalera Ochoa Laura y Castruita Esparza Luis Ubaldo

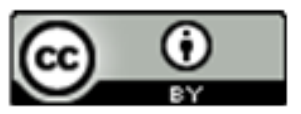

Este texto está protegido por una licencia licencia CreativeCommons 4.0

Usted es libre para Compartir — copiar y redistribuir el $\mathrm{m}$ aterial en cualquier medio o formato-y Adaptar el documento — remezclar, transformar y crear a partir del material- para cualquier propósito, incluso para fines com erciales, siempre que cumpla la condición de:

Atribución: Usted debe dar crédito a la obra original de manera adecuada, proporcionar un enlace a la licencia, e indicar si se han realizado cambios. Puede hacerlo en cualquier forma razonable, pero no de forma tal que sugiera que tiene el apoyo del licenciante o lo recibe por el uso que hace de la obra.

Resumendelicencia - Textocompletodelalicencia 